\title{
Technologie des succédanés de produits laitiers ${ }^{(1)}$
}

\author{
par \\ Jean-Claude MICHEL \\ Présenté à l'occasion du congrès des Technologistes Agricoles
}

La margarine jusqu'à ces dernières années était le seul substitut des produits laitiers. Cependant, au cours des années qui ont suivi la dernière guerre mondiale d'autres produits succédanés furent introduits sur le marché et jouissent maintenant d'une popularité sans cesse croissante (blanchisseurs à café, garnitures fouettées, etc.).

Le consommateur n'est pas tout particulièrement intéressé aux produits succédanés, mais il est désireux d'en acheter lorsque la qualité est satisfaisante et que le prix représente une économie en regard des produits laitiers. Ainsi, le public démontre qu'il n'a pas d'allégeance particulière pour les produits laitiers traditionnels.

Les principaux constituants des produits succédanés sont les mêmes que ceux retrouvés dans les produits laitiers, leur état physique est le même, mais c'est leur origine qui diffère. Tout comme dans le lait, la matière grasse est à l'état d'émulsion, les protéines à l'état de suspension colloïdale et les autres constituants sont dans un état similaire ou en solution, suivant le cas, de manière à ce que les produits succédanés possèdent les mêmes caractéristiques physiques, chimiques et organoleptiques que les produits laitiers qu'ils imitent.

\section{Classification des produits succédanés}

Les produits succédanés des produits laitiers sont classifiés selon leur composition en trois groupes différents.

Les produits modifiés ou réengraissés

Dans ces produits, les protéines proviennent des solides non gras du lait, tandis que la matière grasse est d'origine végétale.

(1) Le Québec Laitier et Alimentaire, mai 1969. 


\section{Les produits synthétiques ou artificiels}

Ce groupe comprend les produits succédanés qui contiennent aucun ingrédient d'origine laitière ou considéré comme tel. Les produits ainsi obtenus sont destinés à ressembler aux produits laitiers et à les remplacer.

\section{Produits intermédiaires}

Dont la composition est intermédiaire entre les deux groupes précédents. C'est le cas de certains blanchisseurs à café.

Le premier groupe et le troisième sont souvent groupés ensemble sous le nom de produits modifiés ou réengraissés.

Ainsi, le terme «succédané » sert à englober et les produits réengraissés et les produits artificiels, lorsqu'on fait allusion à ces deux groupes de produits à la fois.

Voici la liste de certains de ces produits succédanés que l'on rencontre sur le marché :

lait modifié ou réengraissé,

lait synthétique, artificiel ou lait imitation crème modifiée,

blanchisseurs à café, similicrème, poudre à café,

crème fouettée réengraissée,

garniture fouettée ou garniture pour desserts, réengraissées,

crème glacée modifiée, (Mellorine),

crème glacée synthétique,

margarine,

lait évaporé modifić,

lait condensé sucré modifié,

fromage modifié,

crème sure modifiée.

Cette liste des produits succédanés s'allonge rapidement et il est à prévoir que cette tendance se continuera avec les années. Présentement quelques 15 produits succédanés se rencontrent sur le marché et l'évolution du marché américain indique qu'il est possible de fabriquer une vaste gamme de produits de remplacement qui seront acceptés par le consommateur.

J'ai énuméré, précédemment, les principaux constituants des succédanés et voyons maintenant chacun d'eux en considérant les principales caractéristiques qu'ils doivent posséder en fonction du produit fabriqué.

\section{La matière grasse}

La matière grasse utilisée dans la fabrication de ces produits substituts provient des plantes oléagineuses telles que : le coton, la fève soya, le colza, le tournesol et autres.

Les caractéristiques physiques et chimiques de cette matière grasse sont de toute première importance. Le choix de l'huile doit 
être arrêté sur celle qui reproduit le plus fidèlement les caractéristiques de la matière grasse d'origine laitière qu'elle doit remplacer.

Parmi les caractéristiques principales que l'on recherche, mentionnons :

- une saveur douce et stable,

- résistance à l'hydrolyse et à l'oxydation,

- facilité de manipulation et d'entreposage,

- économie et dont l'approvisionnement est facile,

- approvisionnement régulier et facile.

Le contenu en solide de l'huile à différentes températures est une caractéristique importante, tout particulièrement à la température de $92^{\circ} \mathrm{F}$, car il détermine la sensation dégustative produite dans la bouche du consommateur. Egalement le point de fusion à une influence considérable et doit être approprié aux conditions du produit de façon à prévenir cette impression que le produit est graisseux lorsque consommé.

Il est à remarquer que la matière grasse se retrouve dans ces produits succédanés sous forme de fines gouttelettes et qu'ainsi ses caractéristiques organoleptiques sont différentes de celles de son état solide.

Jusqu'à ce jour, l'huile de coco est la plus utilisée en raison des caractéristiques ci-haut mentionnées, mais les recherches se poursuivent en vue de l'utilisation de celle des autres plantes oléagineuses, seules ou en mélange, afin d'obtenir une huile mieux adaptée aux caractéristiques du produit que l'on désire fabriquer ou imiter.

\section{Les protéines}

Les protéines constituent en partie les solides non gras de ces produits et sont, d'origine laitière dans le cas des produits modifiés, ou d'origine végétale dans le cas des produits synthétiques.

La poudre de lait écrémé et la caséine sont les sources de solides non gras d'origine laitière. Pour les produits synthétiques, on utilise du caséinate de sodium ou des extraits de protéines de soya, de coton ou d'autres plantes. Il est à remarquer que la caséine n'est pas considérée comme un produit laitier, au même titre que la poudre de lait, et de ce fait peut être utilisée dans la fabrication des produits synthétiques.

Les protéines servant à la fabrication de ces produits substituts doivent être d'excellente qualité, se dissoudre facilement et complètement, être stable afin de ne pas apporter de saveurs désagréables au produit fini et être une bonne source d'acides aminés essentiels pour l'humain.

Les recherches se poursuivent et s'accentuent en vue de produire économiquement des protéines végétales ayant une excellente qualité nutritive, c'est-à-dire capable de satisfaire complètement les besoins 
de l'homme et ceci à l'aide de mélanges de protéines provenant de différentes plantes et par l'addition de suppléments riches en acides aminés essentiels pour l'homme.

\section{Emulsificateur}

L'agent émulsifiant a pour fonction de favoriser la dispersion de la matière grasse dans la phase aqueuse et de maintenir cette émulsion. Il est un supplément à l'homogénéisation et non un remplacement de celle-ci.

Le choix de l'émulsificateur est fait de façon à donner un produit qui n'est pas trop visqueux, qui s'écoule facilement et qui reste stable pour une période de temps suffisante à sa mise en marché.

Les mono- et di-glycérides sont des agents émulsifiants de choix et leur quantité est établie en fonction du produit et de ses caractéristiques.

\section{L'agent stabilisateur}

Le rôle du stabilisateur dans les produits succédanés est d'améliorer la viscosité du produit par son interaction avec les protéines qu'il maintient en suspension et d'assurer la stabilité du produit au gel et au dégel.

Le choix du stabilisateur se fait en fonction des caractéristiques recherchées dans le produit fini et à partir de la grande variété de gommes comestibles d'origine marine ou végétale, rencontrées sur le marché.

\section{Couleur}

La couleur est très importante, pour assurer le succès de la mise en marché des produits succédanés. C'est pourquoi il est parfois nécessaire d'ajouter un colorant et la Bêta-carotène est communément utilisée pour apporter la teinte désirée dans ces produits.

Il y a deux formes de carotène que l'on peut utiliser :

- La forme liquide, c'est un mélange de B-carotène avec de l'huile végétale dans une proportion de 30 p. 100 de carotène.

- La forme solide, chaque gramme de cette poudre contient $0,010 \mathrm{~g}$ de Bêta-carotène.

Le choix de la forme à utiliser dépend des préférences du transformateur qui désire faire l'apport soit dans la phase aqueuse ou lipidique du produit.

\section{Saveur}

La saveur des produits succédanés est une de leurs caractéristiques de toute première importance et afin de leur donner toute la 
saveur désirée par le consommateur, l'addition de saveur artificielle est souvent souhaitable.

Ces saveurs spécialement préparées pour cette fin se retrouvent facilement sur le marché et ajoutent une grande valeur à ces produits substituts.

\section{Sucres}

Les sucres les plus utilisés dans la fabrication de ces produits succédanés artificiels sont :

- les sirops de maïs à faible équivalent dextrose recherchés en raison de leur douceur, de leur viscosité et de leur pouvoir d'épaississement supérieur.

- parfois, le sucrose ou le lactose sont utilisés pour des raisons d'économie et de commodité.

\section{Vitamines}

Le lait est une source importante en vitamines A, D, E, K et autres qui contribuent à sa haute valeur nutritive. Cependant le lait écrémé est dépourvu des vitamines dites " liposolubles 》 qui vont enrichir la crème telle la vitamine $\mathrm{A}$ par exemple. De plus, en raison des traitements à haute température auxquelles elles sont soumises au cours de leur purification, les huiles végétales sont dépourvues de vitamines.

C'est en vue de palier à cette situation déficitaire que l'on ajoute un supplément de vitamines aux produits succédanés de façon à rencontrer les exigences de l'organisme humain.

Le contrôle de la teneur des succédanés du lait en vitamines ne pose normalement aucun problème car dans certaines régions, le lait naturel est également enrichi en vitamine D.

\section{Minéraux}

Le calcium, le phosphore et le sodium sont les trois principaux minéraux retrouvés dans le lait et constituent souvent l'élément essentiel à l'alimentation des enfants et des vieillards.

Le calcium s'ajoute facilement aux succédanés mais ne se présente pas toujours sous forme assimilable par l'organisme. On peut y remédier en ajoutant de la vitamine D.

Généralement, les aliments qui contiennent du calcium renferment aussi du phosphore. Pour ce qui est du sodium, il peut être ajouté facilement mais doit l'être en quantité proportionnelle aux éléments nutritifs du produit. 